\title{
Primäre Immundefekte erkennen und behandeln
}

Mehr Aufmerksamkeit für eine häufig lange unerkannte Erkrankung und eine bessere Patientenversorgung aber auch Unterstützung der Forschung - das sind die Ziele der US-amerikanisch basierten Jeffrey Modell Stiftung. Im Mittelpunkt stehen primäre Immundefekte, die aufgrund der damit verbundenen zahlreichen schweren Infektionen die Lebensqualität stark beeinträchtigen und mit einem erhöhten Komplikations- und Mortalitätsrisiko verbunden sind. An der Univ.Klinik für Kinder- \& Jugendheilkunde in Wien wurde Ende Jänner das erste Jeffrey Modell Foundation-Zentrum in Österreich mit einem Symposium eröffnet.

Der Forschungsschwerpunkt primäre Immundefekte (pID) wurde bereits vor zehn Jahren an der Medizinischen Universität Wien etabliert und findet sich heute mit dem Jeffrey Modell Diagnostic and Research Center Vienna in der neuen Forschungsplattform Center for Congenital Immunodeficiencies (CCID). Primäre Immundefekte wurden auch neu in den Entwicklungsplan der MedUni Wien aufgenommen. Gemeinsam mit dem St. Anna-Kinderspital betreibt die Univ.-Kinderklinik eine Spezialambulanz, berichtete Univ.-Prof.Dr. Wolfgang Schütz, Rektor der MedUni Wien, bei der Eröffnung.

\section{Seltene Krankheit und hohe Dunkelziffer}

Mit einer Häufigkeit zwischen 1:800 und 1:2000 zählt die heterogene Gruppe der pID zu den Rare Diseases. In Österreich sind 300 Personen mit klinisch relevanten pID in medizinischer Betreuung. Die Dunkelziffer dürfte beträchtlich sein, geben die Experten zu bedenken.

pID stellen eine vielfältige Gruppe von Erkrankungen dar, denen die gestörte Immunfunktion des Organismus gemeinsam ist. Die häufigsten Defekte sind:

- Kombinierte (T- und B-Zell)-Defekte

- Immundefekte, bei denen der Antikörpermangel im Vordergrund steht

- Defekte der Phagozytenzahl und funktion
- Andere gut definierte Immundefekte, wie das Louis-Bar-Syndrom, das DiGeorge-Syndrom oder die chronische mukotane Candidiasis (CMC)

- Das variable Immundefektsyndrom.

Die größte Gruppe von Patienten, mit einem Anteil von mehr als 50 Prozent, weist ein Antikörpermangelsyndrom (AKMS) auf, erklärt Univ.-Prof.Dr. Elisabeth Förster-Waldl vom CCID. „Dabei kommt es durch eine Dysbalance des Immunsystems zu einer fehlenden Toleranz gegenüber dem eigenen Körper." Etwa 70 Prozent der pID-Betroffenen können mit Immunglobulinen gut behandelt werden wenn die Krankheit entsprechend diagnostiziert wurde. Bei einem Teil der übrigen 30 Prozent gibt es, so Förster-Waldl, die Möglichkeit, sowohl Immunglobuline als auch Immunsuppressiva zu verabreichen: „Eine paradoxe Situation“, in der versucht wird, das System durch eine ausgewogene Unterstützung der Abwehr und der Akzeptanz in ein Gleichgewicht zu bringen. Liegt ein sehr schwerer Defekt vor, stellt auch die Knochenmarktransplantation eine Option dar.

Als genetisch bedingte Erkrankung hat die genetische Erforschung und Abklärung des primären Immundefekt (pID) einen bedeutenden Anteil. Mit Hilfe des whole-exom sequencing ist es heute möglich, eine genetische Analyse zu erstellen, um die Grundursache des Defekts zu identifizieren. In nächster Zukunft werden auch auf der Grundlage der Forschungsarbeiten in Wien neue Möglichkeiten für individualisierte Therapien zur Verfügung stehen, berichtet FörsterWaldl. Dann werde mittelfristig auch eine Gentherapie eingesetzt werden können.

\section{pID als Experimente der Natur}

Die Forschung im Bereich der primären Immundefekte ermöglicht auch Erkenntnisse in anderen Zusammenhängen, stellte Dr. Kaan Botzug vom Research Center for Molecular Medicine (CeMM) der MedUni Wien, das ebenfalls am CCID betei- ligt ist, fest und zitierte Prof. Fred D. Rosen, den 2005 verstorbenen Pionier der Immunologie an der Harvard Medical School: „Primäre Immundefekte sind Experimente der Natur, die uns die Immunologie lehren." So versteht man die Erkrankung heute auf molekularer Ebene wesentlich besser, was wiederum zu einem besseren Verständnis der am Immunsystem mitwirkenden Komponenten geführt hat. Es gebe beispielsweise Überlegungen, dass eine der prototypischen Autoimmunerkrankungen, die chronisch entzündlichen Darmerkrankungen, einen primären Immundefekt als Grundlage habe. Dies wiederum habe klinische Konsequenzen. „Die Immunologie steht heute im Zentrum im Verständnis einer großen Zahl an Erkrankungen“, resümierte Botzug

\section{Nächstes Ziel: Neugeborenenscree- ning auf schwere Immundefekte}

Der Status als JMF-Zentrum verleiht den Forschungsaktivitäten in Wien im internationalen Kontext eine besserer Sichtbarkeit. „Die JMF ist extrem erfolgreich und verfügt über ein weltweites Netzwerk das die Bewusstseinsbildung aber auch die Verfügbarkeit von klinischer Labordiagnostik fördert", stellt FörsterWaldl fest. So konnte in den USA 2012 ein flächendeckendes Neugeborenenscreening auf plD etabliert werden - finanziert durch die Jeffrey Modell-Stiftung. Diesbezügliche Bestrebungen gibt es seit einigen Jahren auch in Österreich. Nun hofft man auf einen neuen Impuls.

Die Finanzierung des JMF-Zentrums in Wien ist durch die Firma Octapharma für die kommenden drei Jahre sichergestellt. Damit ist eine wichtige Voraussetzung geschaffen, um die Diagnose und Therapie angeborener Immundefekte in Österreich zu verbessern.

Quelle: Kick-Off Jeffrey Modell Diagnostic and Research Center Vienna, 24. - 25. 1. 2014, Wien

Informationen: www.jmf-vienna.at 